\title{
The relative contribution of intervertebral disc and vertebral wedging to the development of thoracic kyphosis and lumbar lordosis in asymptomatic adolescents
}

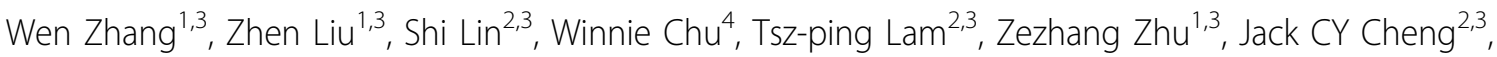 \\ Yong Qiu ${ }^{1,3^{*}}$
}

From The 10th Meeting of the International Research Society of Spinal Deformities (IRSSD 2014 Sapporo) Sapporo, Japan. 29 June - 2 July 2014

\section{Objective}

To analyze the contribution of the intervertebral disc and vertebral wedging to thoracic kyphosis and lumbar lordosis in different growth stages of asymptomatic adolescence.

\section{Methods and materials}

A retrospective study of 170 asymptomatic adolescent volunteers was performed and all of them had the standing whole-spine lateral radiography. All subjects were divided into 3 groups by age: Group A (10-12 years), Group B (13-15 years), and Group C (16-18 years). Four different radiographic parameters were measured: lumbar lordosis (LL, L1-L5), thoracic kyphosis (TK, T4-T12), wedging angle of each disc and vertebra between T4 and L5. In addition, the percent of the sum of T4-T12 disc/vertebral wedging angle in the TK and the sum of the L1-L5 disc/vertebral wedging angle in the LL were calculated.

\section{Results}

The LL increased by $5.1^{\circ}$ from group A to Group C $(\mathrm{P}=0.028)$, followed by an increasing contribution of the lumbar disc wedging from 90.0 percent in Group A to 96.4 percent in Group C $(\mathrm{P}=0.025)$ and a decreasing contribution of the lumbar vertebral wedging from $9.0 \%$ in Group A to 2.9 percent in Group C $(\mathrm{P}=0.02)$. As for the thoracic spine, the TK increased by $5.8^{\circ}$ from Group A to Group $C(p=0.021)$, but no significant differences in the contribution of both disc and vertebral wedging to TK were found among the three groups.

\section{Conclusions}

In the different growth stages of asymptomatic adolescences, an increasing contribution of the disc wedging and a decreasing of the vertebral wedging to the LL are results of age-associated process. However, the contribution of the disc and vertebral wedging to the TK remains stable in different growth periods.

\section{Authors' details \\ 'Spine Surgery, Affiliated Drum Tower Hospital of Nanjing University Medical School, Nanjing 210008, China. ${ }^{2}$ Department of Orthopaedics and \\ Traumatology, Chinese University of Hong Kong, Hong Kong, China. ${ }^{3}$ Joint Scoliosis Research Center of the Chinese University of Hong Kong \& Nanjing University, Nanjing, China. ${ }^{4}$ Department of Radiology, Chinese University of Hong Kong, Hong Kong, China.}

Published: 19 January 2015

doi:10.1186/1748-7161-10-S1-P16

Cite this article as: Zhang et al: The relative contribution of intervertebral disc and vertebral wedging to the development of thoracic kyphosis and lumbar lordosis in asymptomatic adolescents. Scoliosis 2015 10(Suppl 1):P16. 